\title{
A comparative study of microwave assisted and conventional synthesis of 2,3-dihydro-2-aryl-4-[4-(2-oxo-2H-chromen-3-yl)-1,3-thiazol-2- ylamino]-1,5-benzothiazepines and its antimicrobial activity
}

\author{
Jignesh P. Raval,*a Jignasu T. Desai, ${ }^{\mathrm{b}}$ Chintan K. Desai, ${ }^{\mathrm{b}}$ and Kishor R. Desai ${ }^{\mathrm{b}}$ \\ ${ }^{a}$ Ashok \& Rita Patel Institute of Integrated Research in Biotechnology and Allied Sciences, Vallabh \\ vidyanagar, 388120, India \\ ${ }^{b}$ Department of Chemistry, Veer Narmad South Gujarat University, Surat, 395007, India \\ E-mail:dripraval@hotmail.com
}

\begin{abstract}
A variety of 2,3-dihydro-2-aryl-4-[4-(2-oxo-2H-chromen-3-yl)-1,3-thiazol-2-ylamino]-1,5benzothiazipine 6a-j were synthesized from $N$-[4-(2-oxo-2H-chromen-3-yl)-1,3-thiazol-2-yl]cinnamamide derivatives $\mathbf{4 a - j}$. The structures of these compounds were confirmed by IR, NMR $\left({ }^{1} \mathrm{H}\right.$ $\left.\&{ }^{13} \mathrm{C}\right) \&$ mass spectral analysis. A considerable increase in the reaction rate has been observed with better yield using microwave irradiation in comparison to conventional thermal treatment. The newly synthesized compounds were evaluated for antimicrobial activity against variety of bacterial strains and some of these compounds have shown significant antibacterial and antifungal activities.
\end{abstract}

Keywords: $\quad N$-substituted cinnamamides, $\quad N$-[4-(2-oxo-2H-chromen-3-yl)-1,3-thiazol-2-yl] acetamide, 1,5-benzothiazipines, microwave irradiation (MWI), IR, NMR $\left({ }^{1} \mathrm{H} \&{ }^{13} \mathrm{C}\right)$, mass spectral analysis, antibacterial and antifungal activities

\section{Introduction}

The chemistry and pharmacology of thiazoles and thiazolochromenones are of great interest to medicinal chemists nowadays, because they are known to possess a wide range of pharmacological properties $^{1,2}$ including very good antimicrobial, ${ }^{3}$ anti-HIV, ${ }^{4}$ anticoagulant ${ }^{5}$ and anti-allergenic ${ }^{6}$ activities. Similarly, benzothiazipines are well known compounds for diverse therapeutically properties like antimicrobial, ${ }^{7}$ antihypertensive, ${ }^{8}$ calcium channel blocker, ${ }^{9}$ blood platelet aggregation inhibitory ${ }^{10}$ and coronary vasodilatory effects. ${ }^{11}$ As a result, due to their wide range of biological and synthetic applications, various methods have been developed for the synthesis of 1,5benzothiazepines. ${ }^{12,13}$ However, the development of mild and efficient protocols continues to be a challenging endeavor in synthetic organic chemistry. The most straightforward protocol for the synthesis of 1,5-benzothiazipine involves the condensation of chalcones with 2aminobenzenethiols in methanol/ethanol under strong acidic conditions. ${ }^{14}$ 
Earlier, we reported on the microwave-induced Niementowskii reaction; synthesis of quinazolinones and 3-methyl-1H-5-pyrazolones using different solid supports, ${ }^{15}$ and fluorine containing pyrozoline derivatives over solid potassium carbonate, ${ }^{16}$ wherein the problems associated with prolonged heating were avoided. We have also successfully adopted this technique for the synthesis of various 1,5-benzothiazipine derivatives. ${ }^{17}$ The advantages of microwave technology over conventional methods in heterocyclic synthesis have been recently reviewed. ${ }^{18}$ For conventional methodology, the yield is sometimes lower than that obtained by microwave protocols.

In view of the above and in continuation to our earlier work ${ }^{19,20}$ on the application of MORE ${ }^{20-24}$ chemistry to organic synthesis, we now report a simple microwave synthesis of 2,3-dihydro-2-aryl4-[4-(2-oxo-2H-chromen-3-yl)-1,3-thiazol-2-ylamino]-1,5-benzothiazipine 6a-j. By successfully incorporating benzothiazipines with thiazolochromenones novel, pharmacologically active compounds were obtained. All compounds were prepared using both conventional and microwave techniques. The reaction carried out in methanol using conventional thermal treatment required about 6-9 hr while microwave irradiation required only $1.5-3 \mathrm{~min} .{ }^{13}$ For the conventional method, the yield is lower in comparison to microwave irradiation. A comparative study in terms of yield and reaction period is shown in Table 1.

\section{Results and Discussion}

\section{Chemistry}

$N$-Substituted cinnamamides $\mathbf{4 a - j}$ derived of $N$-[4-(2-oxo-2H-chromen-3-yl)-1,3-thiazol-2yl]acetamide 2 were obtained using various aldehydes 3a-j. $N$-[4-(2-oxo-2H-chromen-3-yl)-1,3thiazol-2-yl]acetamide 2 was itself prepared from 3-(2-amino-1,3-thiazol-4-yl)-2H-chromen-2-one 1 by acetylation with acetyl chloride in chloroform. Further, 4a-j on treatment with 2 -aminobenezene thiol 5 in the presence of glacial acetic acid as catalyst and methanol as reaction mediator afforded 2,3-dihydro-2-aryl-4-[4-(2-oxo-2H-chromen-3-yl)-1,3-thiazol-2-ylamino]-1,5-benzothiazipine 6a-j (Scheme 1).

Formation of $N$-substituted cinnamamides $\mathbf{4 a - j}$ was evidenced by appearance of a signal at 6.9$7.2 \delta \mathrm{ppm}$ due to $-\mathrm{CH}=\mathrm{CH}$ - and IR bands at $1705-1682 v_{\max } \mathrm{cm}^{-1}$ stretching, due to the $>\mathrm{C}=\mathrm{O}$ of the $\alpha, \beta$ unsaturated chalcone. Furthermore, signals in the ${ }^{1} \mathrm{H}$ NMR spectra of 1,5-benzothiazepines $\mathbf{6 a}-\mathbf{j}$, revealed doublets at $3.93(\mathrm{~J}=6.8 \mathrm{~Hz}) \delta \mathrm{ppm}$ due to $-\mathrm{CH}_{2}-$ and triplets at $3.30(\mathrm{~J}=7.0 \mathrm{~Hz}) \delta$ ppm due to $-\mathrm{CH}-$. The deshielded absorption of the $\mathrm{CH}$ proton is explained by its attachment to the electronegative sulfur, a phenyl group and a carbon atom having $2 \mathrm{H}$ atoms. Similarly in the ${ }^{13} \mathrm{C}$ NMR spectra signals were observed in the range of $123.07-125.07 \delta \mathrm{ppm}$ due to $-\mathrm{CH}_{2}-$ and in the range of $60.17-60.97 \delta \mathrm{ppm}$ due to $-\mathrm{CH}-$. In the IR of 1,5-benzothiazepine $\mathbf{6 a - j}$, bands at $3270-$ $3261 v_{\max } \mathrm{cm}^{-1}$ (stretching, $-\mathrm{CH}-$ ), $1609-1607 v_{\max } \mathrm{cm}^{-1}$ (stretching, $\mathrm{C}=\mathrm{N}$ ) and in the range of 765 $-635 v_{\max } \mathrm{cm}^{-1}$ (stretching, C-S) confirm the formation of 1,5-benzothiazepines $\mathbf{6 a - j}$. 
<smiles>Cc1csc(-c2cc3ccccc3oc2=O)n1</smiles>

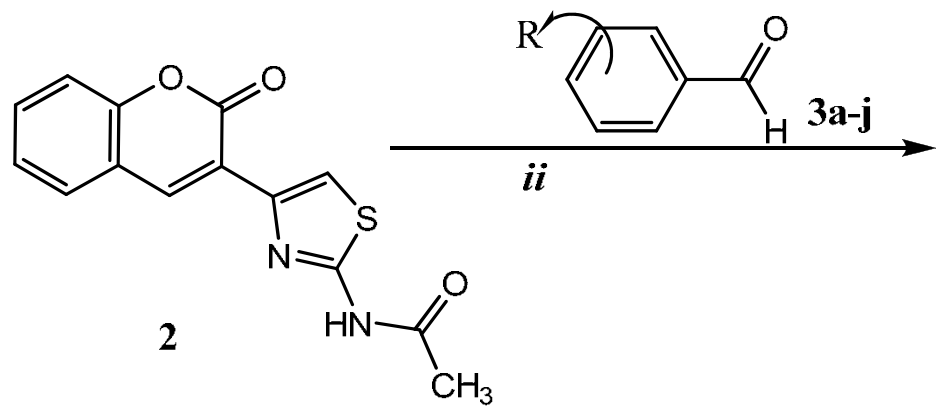
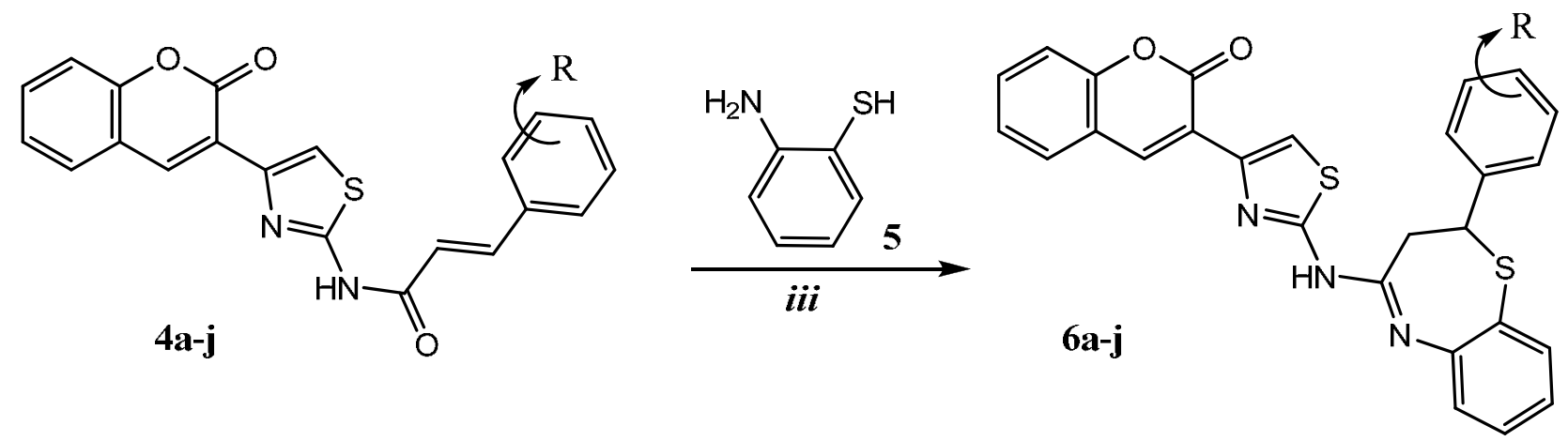

$\mathbf{R}=$ a: $3-\mathrm{CH}_{3},-4-\mathrm{OH} ;$ b: $4-\mathrm{CH}_{3}$; c: 2-Cl; d: 4-Cl; e: 2,4-(Cl) $;$; f: 2-OCH ; g: 4- $-\mathrm{OCH}_{3}$; h: $3,4,5-\left(\mathrm{OCH}_{3}\right)_{3} ;$ i: $4-\mathrm{N}\left(\mathrm{CH}_{3}\right)_{2}$; j: $4-\mathrm{OH}$

Scheme 1. Reagents and reaction conditions: (i) $\mathrm{CH}_{3} \mathrm{COCl} / \mathrm{CHCl}_{3}, 0-5{ }^{\circ} \mathrm{C}, 8 \mathrm{hr}$. (ii) Conventional: $\mathrm{CH}_{3} \mathrm{OH} / 2 \% \mathrm{NaOH}$, Reflux, 6-8 hr. Microwave: $\mathrm{CH}_{3} \mathrm{OH} / 2 \% \mathrm{NaOH}$, MWI, 2.0-3.5 min. (iii) Conventional: $\mathrm{CH}_{3} \mathrm{OH} /$ glacial $\mathrm{CH}_{3} \mathrm{COOH}$, Reflux, 60-70 ${ }^{\circ} \mathrm{C}$, 6-9 hr. Microwave: $\mathrm{CH}_{3} \mathrm{OH} /$ glacial $\mathrm{CH}_{3} \mathrm{COOH}$, MWI, 2.0-3.5 min.

All the reactions under MWI were completed within 2-3.5 min whereas similar reactions under conventional heating (steam bath) at reflux gave poorer yields after much longer reaction times (Table 1).

The impact of microwave irradiation and conventional heating for the synthesis of compounds 4a-j and $\mathbf{6 a}-\mathbf{j}$ has been compared. Moreover the effects of irradiation power and time on the reaction were also studied and the results are summarized in Table 1. It was found that the higher yields of compounds $\mathbf{4 a}-\mathbf{j}$ and $\mathbf{6 a}-\mathbf{j}$ were obtained at 500 watt for $2-2.5$ min of microwave irradiation. The structures of the above compounds were in good agreement with obtained spectral and analytical data. 
Table 1. Comparison of Conventional and Microwave synthesis for $4 \mathbf{a} \mathbf{j}$ and $\mathbf{6 a}-\mathbf{j}$

\begin{tabular}{cccccccc}
\hline Compd. & \multicolumn{6}{c}{ Conventional } & \multicolumn{5}{c}{ Microwave } \\
\cline { 2 - 8 } & \% yield & $\mathrm{t} / \mathrm{hrs}$ & \% yield & $\mathrm{t}_{1} /$ min & $\mathrm{P}_{1} /$ watt & $\mathrm{t}_{2} /$ min & $\mathrm{p}_{2} /$ watt \\
\hline $\mathbf{4 a}$ & 61 & 6 & 82 & 3.5 & 350 & 2.0 & 500 \\
$\mathbf{4 b}$ & 58 & 6 & 86 & 3.0 & 350 & 2.0 & 500 \\
$\mathbf{4 c}$ & 63 & 6.5 & 84 & 3.5 & 350 & 2.5 & 500 \\
$\mathbf{4 d}$ & 66 & 7 & 87 & 3.5 & 350 & 2.0 & 500 \\
$\mathbf{4 e}$ & 67 & 7 & 88 & 3.5 & 350 & 2.5 & 500 \\
$\mathbf{4 f}$ & 68 & 7 & 89 & 3.5 & 350 & 2.0 & 500 \\
$\mathbf{4 g}$ & 69 & 8 & 81 & 3.0 & 350 & 2.5 & 500 \\
$\mathbf{4 h}$ & 70 & 8 & 86 & 3.5 & 350 & 2.0 & 500 \\
$\mathbf{4 i}$ & 71 & 6.5 & 88 & 3.0 & 350 & 2.0 & 500 \\
$\mathbf{4 j}$ & 72 & 6 & 87 & 3.5 & 350 & 2.5 & 500 \\
$\mathbf{6 a}$ & 73 & 7 & 88 & 3.5 & 350 & 2.5 & 500 \\
$\mathbf{6 b}$ & 77 & 7 & 92 & 3.0 & 350 & 2.0 & 500 \\
$\mathbf{6 c}$ & 71 & 8 & 91 & 3.0 & 350 & 2.0 & 500 \\
$\mathbf{6 d}$ & 72 & 8 & 92 & 3.5 & 350 & 2.5 & 500 \\
$\mathbf{6 e}$ & 68 & 6 & 88 & 3.5 & 350 & 2.0 & 500 \\
$\mathbf{6 f}$ & 69 & 7 & 85 & 3.5 & 350 & 2.0 & 500 \\
$\mathbf{6 g}$ & 71 & 9 & 87 & 3.5 & 350 & 2.0 & 500 \\
$\mathbf{6 h}$ & 66 & 9 & 88 & 3.5 & 350 & 2.5 & 500 \\
$\mathbf{6 i}$ & 65 & 8 & 89 & 3.5 & 350 & 2.5 & 500 \\
$\mathbf{6 j}$ & 72 & 8 & 92 & 3.5 & 350 & 2.0 & 500 \\
\hline
\end{tabular}

\section{Antimicrobial activity}

All the newly synthesized compounds were screened in vitro for their antimicrobial activity using the streak plate and cup plate method by measuring the zone of inhibition according to a standard procedure ${ }^{25}$ against a variety of bacterial strains such as gram $+v e$ bacteria [Bacills substilis (ATCC6633) and Staphylococcus aureus (ATCC-6538)] and gram -ve bacteria [Escherchia coli (ATCC6538), Pseudomonas aerugina (ATCC-1539)]. In addition, some fungal pathogens [Candida albicans (ATCC-64550), Candida krusei (ATCC-14243) and Candida parapsilosis (ATCC-22019)] (Table 2) were also tested. Sensitivity of the selected microorganisms to some synthesized compounds $4 \mathbf{a}-\mathbf{j}$ and $\mathbf{6 a}-\mathbf{j}$ was determined in vitro at a concentration of $100 \mu \mathrm{g} / \mathrm{mL}$ in $\mathrm{CHCl}_{3}$. Standard drugs Ampicillin, Amoxicillin, Penicillin and Flucanozole were also screened under similar conditions for comparison. By visualizing the antimicrobial data it could be observed that some of the compounds possess significant activity. However, the activities of the tested compounds are less than that of standard antibacterial and antifungal agents. Results are presented in Table 2. 
Table 2. Antimicrobial activity $(100 \mu \mathrm{g} / \mathrm{ml})$ of Compound $\mathbf{4 a - j}$ and $\mathbf{5 a - j}$

\begin{tabular}{|c|c|c|c|c|c|c|c|}
\hline \multirow{3}{*}{ Compound } & \multicolumn{4}{|c|}{ Antibacterial activity } & \multirow{2}{*}{\multicolumn{3}{|c|}{ Antifungal activity }} \\
\hline & \multicolumn{2}{|c|}{ Gram +ve } & \multicolumn{2}{|c|}{ Gram - ve } & & & \\
\hline & $B . s^{\mathrm{a}}$ & S. $a^{\mathrm{b}}$ & $E . c^{\mathrm{c}}$ & $P \cdot a^{\mathrm{d}}$ & C. $a^{\mathrm{e}}$ & C. $k^{\mathrm{f}}$ & C. $p^{\mathrm{g}}$ \\
\hline $4 a$ & +++ & ++ & + & +++ & ++ & ++ & + \\
\hline 4b & ++ & ++ & ++ & ++ & + & + & + \\
\hline 4c & +++ & ++ & +++ & +++ & ++ & ++ & ++ \\
\hline 4d & ++ & ++ & ++ & ++ & + & + & + \\
\hline $4 e$ & + & ++ & ++ & ++ & + & ++ & ++ \\
\hline 4f & ++ & ++ & +++ & + & + & - & + \\
\hline $4 g$ & ++ & ++ & ++ & ++ & ++ & ++ & ++ \\
\hline $4 h$ & ++ & ++ & ++ & ++ & ++ & ++ & ++ \\
\hline $4 \mathbf{i}$ & ++ & + & ++ & ++ & - & ++ & ++ \\
\hline $4 j$ & +++ & +++ & +++ & +++ & ++ & ++ & ++ \\
\hline $6 a$ & ++ & + & ++ & +++ & +++ & ++ & ++ \\
\hline $6 b$ & +++ & ++ & ++ & +++ & ++ & +++ & ++ \\
\hline $6 c$ & ++ & ++ & + & ++ & ++ & ++ & ++ \\
\hline 6d & +++ & ++ & +++ & +++ & +++ & +++ & ++ \\
\hline $6 e$ & ++ & ++ & +++ & +++ & +++ & +++ & +++ \\
\hline $6 f$ & +++ & +++ & ++ & +++ & +++ & ++ & +++ \\
\hline $6 g$ & +++ & +++ & ++ & +++ & ++ & ++ & ++ \\
\hline $6 h$ & ++ & ++ & ++ & ++ & +++ & +++ & ++ \\
\hline $6 \mathbf{i}$ & ++ & ++ & ++ & ++ & ++ & ++ & ++ \\
\hline $6 \mathbf{j}$ & ++ & ++ & ++ & ++ & ++ & ++ & ++ \\
\hline Ampicillin & ++++ & ++++ & +++++ & ++++ & & & \\
\hline Amoxicillin & ++++ & +++++ & ++++ & +++++ & & & \\
\hline Penicillin & ++++ & +++ & +++++ & ++++ & & & \\
\hline Flucanozole & & & & & +++++ & ++++ & ++++ \\
\hline
\end{tabular}

${ }^{\mathrm{a}} B . s$ (ATCC-6633) - Bacills substilis. ${ }^{\mathrm{b}} S . a(\mathrm{ATCC}-6538)-$ Staphylococcus aureus. ${ }^{\mathrm{c}} E$. $c$ (ATCC6538) - Escherchia coli. ${ }^{\mathrm{d}}$ P. a (ATCC-1539) - Pseudomonas aeruginosa. ${ }^{\mathrm{e}}$ C. a (ATCC-64550) Candida albicans. ${ }^{\mathrm{f}} C . k$ (ATCC-14243) - Candida krusei. ${ }^{\mathrm{g}} C . p$ (ATCC-22019) - Candida parapsilosis.

\section{Conclusions}

A new method for the synthesis of benzothiazipine derivatives using microwave irradiation, offers significant improvements over existing procedures and thus helps facile entry into a synthesis of a variety of 1,5-benzothiazipines, of potentially high synthetic utility. Also, this simple and 
reproducible technique affords various 1,5-benzothiazipine derivatives with short reaction times, excellent yields, and without formation of undesirable side products.

\section{Experimental Section}

General Procedures. Melting points were determined using a PMP - DM scientific melting point apparatus and are uncorrected. The purity of the compounds was checked by TLC $(0.5 \mathrm{~mm}$ thickness) using silica gel-G coated Al-plates (Merck) and spots were visualized by exposing the dry plates to iodine vapors. IR spectra $\left(v_{\max }\right.$ in $\left.\mathrm{cm}^{-1}\right)$ were recorded on a Perkin Elmer spectrum BX series FT-IR spectrometer using $\mathrm{KBr}$ or Nujol technique. NMR spectra were recorded on a Bruker WM 400 FT MHz NMR instrument using $\mathrm{CDCl}_{3}$ or $\mathrm{DMSO}-d_{6}$ as solvent and TMS as internal reference (chemical shifts in $\delta$, ppm). Mass spectra were run on a Varian model MAT MS-311 spectrometer at $70 \mathrm{eV}$. The elemental analysis $(\mathrm{C}, \mathrm{H}, \mathrm{N})$ of compounds were performed at SAIF, Central Instrumentation laboratory, Punjab University, Chandigrah (India) using a Carlo Erba-1108 elemental analyzer. Results were found to be in good agreement with the calculated values. The microwave assisted reactions are conducted in a "QPro-M Modified Microwave Synthesis System" manufactured by Questron Technologies Corporation, Ontario L4Z 2E9 Canada, whereby microwaves are generated by magnetron at a frequency of $2450 \mathrm{MHz}$ having an output energy range of 100 to 500 Watts and with an individual sensor for temperature control (fiber optic is used as a individual sensor for temperature control) with attachment of reflux condenser with constant stirring (thus avoiding the risk of high pressure development) and synthesis on preparative scales.

3-(2-Amino-1,3-thiazol-4-yl)-2H-chromen-2-one (1). The starting compound was synthesized from the reaction of 3-bromoacetylcoumarin with thiourea. ${ }^{26}$

Synthesis of $\mathrm{N}$-[4-(2-oxo-2H-chromen-3-yl)-1,3-thiazol-2-yl]acetamide (2). A solution of 2amino-4-(coumarin-3-yl)-thiazole (1) (2.44 g, $0.01 \mathrm{~mol})$ was taken up in chloroform (50 $\mathrm{mL})$ and acetylchloride $(0.78 \mathrm{~g}, 0.01 \mathrm{~mol})$ was added drop wise with constant stirring at $0-5{ }^{\circ} \mathrm{C}$. The reaction mixture was stirred for $8 \mathrm{hr}$. The solvent was distilled off and the solid product was filtered, dried and recrystallized from absolute alcohol. Anal. Calcd. for $\mathrm{C}_{11} \mathrm{H}_{11} \mathrm{NO}_{2} \mathrm{~S}_{2}$ : C, 52.18; H, 4.22; N, 5.40. Found: C, 52.16; H, 4.23; N, 5.38\%; yield 71\%, m.p. $185{ }^{\circ} \mathrm{C}$. IR [v, $\left.\mathrm{cm}^{-1}, \mathrm{KBr}\right]: 3023$ (aromatic ring), $1675\left(\mathrm{NHCOCH}_{3}\right)$, and $1614(\mathrm{C}=\mathrm{N}), \quad 638(\mathrm{C}-\mathrm{S}), \quad 1723\left(>\mathrm{C}=\mathrm{O}\right.$ of coumarine); ${ }^{1} \mathrm{H} \mathrm{NMR}$ [400MHz, $\left.\delta, \mathrm{ppm}, \mathrm{DMSO}-d_{6}\right]: 6.73-7.87(\mathrm{~m}, 6 \mathrm{H}, \mathrm{ArH}), 5.18\left(\mathrm{~s}, 1 \mathrm{H}, \mathrm{NH}\right.$ of amide), $1.56\left(\mathrm{~s}, 3 \mathrm{H}, \mathrm{CH}_{3}\right.$ of amide).

\section{General procedure of $\boldsymbol{N}$-[4-(2-oxo-2H-chromen-3-yl)-1,3-thiazol-2-yl]cinnamamides (4a-j) $)^{27}$}

(a) Conventional method. A solution of 2- $N$-acetyl-4-(coumarin-3-yl)-thiazole (2) (2.86 g, 0.01 mole) in methanol $(30 \mathrm{~mL})$ and various aromatic aldehydes $(3 \mathbf{a}-\mathbf{j})(0.01 \mathrm{~mol})$ were taken and to it 5 $\mathrm{mL}$ of $2 \% \mathrm{NaOH}$ solution was added. The reaction mixture refluxed for 6-8 $\mathrm{hr}$ and then the solvent was removed by vacuum distillation. The solid product was filtered, dried and recrystalised from absolute alcohol. Completion of the reaction was monitored by TLC. 
(b) Microwave method. A solution of 2- $N$-acetyl-4-(coumarin-3-yl)-thiazole (2.86 g, $0.01 \mathrm{~mol}$ ) (2) in methanol $(30 \mathrm{~mL})$ and various aromatic aldehydes $(\mathbf{3 a - j})(0.01 \mathrm{~mol})$ were taken and to it $5 \mathrm{~mL}$ of $2 \% \mathrm{NaOH}$ solution was added. The reaction mixture was taken in round-bottomed flask placed in a microwave oven and irradiated for 2.0-3.5 min. and then the solvent was removed by vacuum distillation. The solid product was filtered, dried and recrystallized from absolute alcohol.

$\mathrm{N}$-[4-(2-Oxo-2H-chromen-3-yl)-1,3-thiazol-2-yl]-3-methoxy-4-hydroxycinnamamide (4a). M.p. 137-138 ${ }^{\circ} \mathrm{C}$; IR [v, cm $\left.{ }^{-1}, \mathrm{KBr}\right]: 3450(\mathrm{OH}), 3267(\mathrm{C}-\mathrm{H}), 3025$ (aromatic ring), $2830\left(\mathrm{OCH}_{3}\right)$, 1723 $(>\mathrm{C}=\mathrm{O}$, coumarin), 1705(stretching band $>\mathrm{C}=\mathrm{O}, \alpha, \beta$-unsaturated cinnamamide), $1609(\mathrm{C}=\mathrm{N})$, 1545(-NH), 635(C-S). ${ }^{1} \mathrm{H}$ NMR [400MHz, $\delta$, ppm, DMSO- $d_{6}$ ]: 7.13-7.87(m,6H, ArH), 6.9$7.0(\mathrm{~s}, 1 \mathrm{H}, \mathrm{CH}=\mathrm{CH}), \quad 5.21(\mathrm{~s}, 1 \mathrm{H},-\mathrm{NH}), \quad 4.08(\mathrm{~d}, 1 \mathrm{H},-\mathrm{OH}), \quad 3.61\left(\mathrm{~d}, 3 \mathrm{H},-\mathrm{OCH}_{3}\right)$. Anal. Calcd. for $\mathrm{C}_{22} \mathrm{H}_{16} \mathrm{~N}_{2} \mathrm{O}_{5} \mathrm{~S}: \mathrm{C}, 60.69 ; \mathrm{H}, 3.60 ; \mathrm{N}, 6.50$. Found: C, 62.88; H, 3.77; N, 6.69\%.

N-[4-(2-Oxo-2H-chromen-3-yl)-1,3-thiazol-2-yl]-4-methylcinnamamide (4b). M.p. 143-144 ${ }^{\circ} \mathrm{C}$; IR $\left[v, \mathrm{~cm}^{-1}, \mathrm{KBr}\right]: 3270(\mathrm{C}-\mathrm{H}), 3027$ (aromatic ring), 2928( $\left.\mathrm{CH}_{3}\right), 1722(>\mathrm{C}=\mathrm{O}$, coumarin), 1708 (stretching band $>\mathrm{C}=\mathrm{O}, \alpha, \beta$-unsaturated cinnamamide), $1609(\mathrm{C}=\mathrm{N}), 1542(\mathrm{NH}), 632(\mathrm{C}-\mathrm{S}) .{ }^{1} \mathrm{H}$ NMR [400MHz, $\delta$, ppm, DMSO- $\left.d_{6}\right]$ : 7.11-7.89(m, 7H, ArH), 6.9-7.0(s,1H,CH=CH), 5.23(s,1H, -NH), 2.65(d, 3H,- $\left.-\mathrm{CH}_{3}\right)$. Anal. Calcd. for $\mathrm{C}_{22} \mathrm{H}_{16} \mathrm{~N}_{2} \mathrm{O}_{3} \mathrm{~S}: \mathrm{C}, 67.73 ; \mathrm{H}, 3.11 ; \mathrm{N}, 6.34$. Found: C, 67.61; H, $3.00 ; \mathrm{N}, 6.14 \%$.

$\boldsymbol{N}$-[4-(2-Oxo-2H-chromen-3-yl)-1,3-thiazol-2-yl]-2-chlorocinnamamide (4c). M.p. $167-168{ }^{\circ} \mathrm{C}$; IR $\left[\mathrm{v}, \mathrm{cm}^{-1}, \mathrm{KBr}\right]: 3256(\mathrm{C}-\mathrm{H}), 3023$ (aromatic ring), 1725( $>\mathrm{C}=\mathrm{O}$, coumarin), 1713 (stretching band $>\mathrm{C}=\mathrm{O}, \alpha, \beta$-unsaturated cinnamamide $), 1612(\mathrm{C}=\mathrm{N}), 1542(\mathrm{NH}), 810(\mathrm{C}-\mathrm{Cl}), 638(\mathrm{C}-\mathrm{S}) .{ }^{1} \mathrm{H} \mathrm{NMR}$ [400MHz, $\delta$, ppm, DMSO-d $]$ ]: 7.15-7.97(m, 7H, ArH), 6.9-7.0(s,1H,CH=CH), 5.25(s, 1H, -NH). Anal. Calcd. for $\mathrm{C}_{21} \mathrm{H}_{13} \mathrm{~N}_{2} \mathrm{O}_{3} \mathrm{SCl}$ : C, 60.68; H, 3.18; N, 6.85. Found: C, 60.40; H, 3.10; N, 6.72\%.

$\boldsymbol{N}$-[4-(2-oxo-2H-chromen-3-yl)-1,3-thiazol-2-yl]-4-chlorocinnamamide (4d). M.p. $171-178{ }^{\circ} \mathrm{C}$; IR $\left[v, \mathrm{~cm}^{-1}, \mathrm{KBr}\right]: 3265(\mathrm{C}-\mathrm{H}), 3028$ (aromatic ring), 1721( $>\mathrm{C}=\mathrm{O}$, coumarin), $1613(\mathrm{C}=\mathrm{N}), 1702$ (stretching band $>\mathrm{C}=\mathrm{O}, \alpha, \beta$-unsaturated cinnamamide), 1548(NH), 817(C-Cl), 634(C-S). ${ }^{1} \mathrm{H}$ NMR [400MHz, $\delta$, ppm, DMSO-d $d_{6}$ : 7.10-7.95(m, 7H, ArH), 6.9-7.0(s, 1H, CH=CH), 5.27(s, 1H, -NH). Anal. Calcd. for $\mathrm{C}_{21} \mathrm{H}_{13} \mathrm{~N}_{2} \mathrm{O}_{3} \mathrm{SCl}$ : C, 60.68; H, 3.18; N, 6.85. Found: C, 61.60; H, 3.09; N, 6.77\%. $\boldsymbol{N}$-[4-(2-oxo-2H-chromen-3-yl)-1,3-thiazol-2-yl]-2,4-dichlorocinnamamide (4e). M.p. 193-194 ${ }^{\circ} \mathrm{C}$; IR [v, $\left.\mathrm{cm}^{-1}, \mathrm{KBr}\right]: 3270(\mathrm{C}-\mathrm{H}), 3024$ (aromatic ring), $1723(>\mathrm{C}=\mathrm{O}$, coumarin), $1610(\mathrm{C}=\mathrm{N})$, 1706(stretching band $>\mathrm{C}=\mathrm{O}, \alpha, \beta$-unsaturated cinnamamide), $1550(\mathrm{NH}), 818(\mathrm{C}-\mathrm{Cl}), 636(\mathrm{C}-\mathrm{S}) .{ }^{1} \mathrm{H}$ NMR [400MHz, $\delta$, ppm, DMSO-d $d_{6}$ : 7.14-7.89(m, 6H, ArH), 6.9-7.0(s, 1H, CH=CH), $5.26(\mathrm{~s}, 1 \mathrm{H},-$ $\mathrm{NH}$ ). Anal. Calcd. for $\mathrm{C}_{21} \mathrm{H}_{12} \mathrm{~N}_{2} \mathrm{O}_{3} \mathrm{SCl}_{2}$ : C, 56.88; H, 2.70; N, 6.32. Found: C, 56.42; H, 2.49; N, $6.29 \%$.

N-[4-(2-Oxo-2H-chromen-3-yl)-1,3-thiazol-2-yl]-2-methoxycinnamamide (4f). M.p. $182-181{ }^{\circ} \mathrm{C}$; IR $\left[v, \mathrm{~cm}^{-1}, \mathrm{KBr}\right]: 3267(\mathrm{C}-\mathrm{H}), \quad 3029($ aromatic $\operatorname{ring}), \quad 2830\left(\mathrm{OCH}_{3}\right), 1722(>\mathrm{C}=\mathrm{O}$, coumarin), $1611(\mathrm{C}=\mathrm{N}), 1705$ (stretching band $>\mathrm{C}=\mathrm{O}, \alpha, \beta$-unsaturated cinnamamide), $1545(\mathrm{NH}), 637(\mathrm{C}-\mathrm{S}) .{ }^{1} \mathrm{H}$ NMR [400MHz, $\delta$, ppm, DMSO- $\left.d_{6}\right]: 7.16-7.89(\mathrm{~m}, 7 \mathrm{H}, \mathrm{ArH}), 6.9-7.0(\mathrm{~s}, 1 \mathrm{H}, \mathrm{CH}=\mathrm{CH}), 5.21(\mathrm{~s}, 1 \mathrm{H},-$ $\mathrm{NH})$, 3.63(s, 3H, $\left.-\mathrm{OCH}_{3}\right)$. Anal. Calcd. for $\mathrm{C}_{22} \mathrm{H}_{16} \mathrm{~N}_{2} \mathrm{O}_{4} \mathrm{~S}: \mathrm{C}, 65.34 ; \mathrm{H}, 3.96 ; \mathrm{N}$, 6.93. Found: C, $64.71 ; \mathrm{H}, 3.79 ; \mathrm{N}, 6.71 \%$.

$\boldsymbol{N}$-[4-(2-Oxо-2H-chromen-3-yl)-1,3-thiazol-2-yl]-4-methoxycinnamamide (4g). M.p. 168$169^{\circ} \mathrm{C}$; IR [v, $\left.\mathrm{cm}^{-1}, \mathrm{KBr}\right]:$ 3261 $(\mathrm{C}-\mathrm{H}), 3027$ (aromatic ring), 2837( $\left.\mathrm{OCH}_{3}\right), 1727(>\mathrm{C}=\mathrm{O}$, coumarin), 
1607 $(\mathrm{C}=\mathrm{N}), 1710$ (stretching band $>\mathrm{C}=\mathrm{O}, \alpha, \beta$-unsaturated cinnamamide), $1540(\mathrm{NH}), 633(\mathrm{C}-\mathrm{S}) .{ }^{1} \mathrm{H}$ NMR [400MHz, $\left.\delta, \mathrm{ppm}, \mathrm{DMSO}-d_{6}\right]:$ 7.14-7.89(m,7H, ArH), 6.9-7.0(s, $\left.1 \mathrm{H}, \mathrm{CH}=\mathrm{CH}\right), 5.23(\mathrm{~s}, 1 \mathrm{H},-$ $\mathrm{NH}), 3.66\left(\mathrm{~d}, 3 \mathrm{H},-\mathrm{OCH}_{3}\right)$. Anal. Calcd. for $\mathrm{C}_{22} \mathrm{H}_{16} \mathrm{~N}_{2} \mathrm{O}_{4} \mathrm{~S}: \mathrm{C}, 65.34 ; \mathrm{H}, 3.96 ; \mathrm{N}$, 6.93. Found: $\mathrm{C}$, 65.07; H, 3.69; N, 6.88\%.

N-[4-(2-Oxo-2H-chromen-3-yl)-1,3-thiazol-2-yl]-3,4,5-trimethoxycinnamamide (4h). M.p. 200$201{ }^{\circ} \mathrm{C}$; IR [v, cm ${ }^{-1}, \mathrm{KBr}$ ]: $3265(\mathrm{C}-\mathrm{H}), 3028$ (aromatic ring), 2838( $\left.\mathrm{OCH}_{3}\right), 1723(>\mathrm{C}=\mathrm{O}$, coumarin), $1612(\mathrm{C}=\mathrm{N}), 1703$ (stretching band $>\mathrm{C}=\mathrm{O}, \alpha, \beta$-unsaturated cinnamamide), 1542(NH), 637(C-S). ${ }^{1} \mathrm{H}$ NMR [400MHz, $\left.\delta, \mathrm{ppm}, \mathrm{DMSO}-d_{6}\right]:$ 7.16-7.92(m, 7H, ArH), 6.9-7.0(s,1H, CH=CH), 5.27(s,1H, $\mathrm{NH}), 4.12(\mathrm{~d}, 1 \mathrm{H},-\mathrm{OH}), 3.66\left(\mathrm{~s}, 3 \mathrm{H},-\mathrm{OCH}_{3}\right)$. Anal. Calcd. for $\mathrm{C}_{24} \mathrm{H}_{20} \mathrm{~N}_{2} \mathrm{O}_{6} \mathrm{~S}: \mathrm{C}, 62.06 ; \mathrm{H}, 4.31 ; \mathrm{N}$, 6.03. Found: C, 61.71; H, 4.00; N, 6.14\%.

$\boldsymbol{N}$-[4-(2-Oxо-2H-chromen-3-yl)-1,3-thiazol-2-yl]-4-(dimethylamino)cinnamamide (4i). M.p. 187-88 ${ }^{\circ} \mathrm{C}$; IR $\left[\mathrm{v}, \mathrm{cm}^{-1}, \mathrm{KBr}\right]: 3271(\mathrm{C}-\mathrm{H}), 3024$ (aromatic ring), 1727 ( $>\mathrm{C}=\mathrm{O}$, coumarine), 1709 (stretching band $>\mathrm{C}=\mathrm{O}, \alpha, \beta$-unsaturated cinnamamide), $1544(\mathrm{NH}), 1612(\mathrm{C}=\mathrm{N}), 1319\left(\mathrm{~N}_{(}\left(\mathrm{CH}_{3}\right)_{2}\right)$, 635(C-S). ${ }^{1} \mathrm{H}$ NMR $\left[400 \mathrm{MHz}, \delta\right.$, ppm, DMSO- $\left.d_{6}\right]$ : 7.16-7.87(m, 7H, ArH), 6.9-7.0(s,1H, CH=CH), 5.29(s,1H,-NH), 4.10(d,1H,-OH), 2.92(d,6H,-N( $\left.\left.\mathrm{CH}_{3}\right)_{2}\right)$. Anal. Calcd. for $\mathrm{C}_{23} \mathrm{H}_{19} \mathrm{~N}_{3} \mathrm{O}_{3} \mathrm{~S}: \mathrm{C}, 66.18$; H, 4.55; N, 10.07. Found: C, 66.09; H, 4.61; N, 10.00\%.

N-[4-(2-Oxo-2H-chromen-3-yl)-1,3-thiazol-2-yl]-4-hydroxycinnamamide (4j). M.p. $157-58{ }^{\circ} \mathrm{C}$; IR $\left[v, \mathrm{~cm}^{-1}, \mathrm{KBr}\right]: 3456(\mathrm{OH}), 3272(\mathrm{C}-\mathrm{H}), 3027$ (aromatic ring), 1725( $>\mathrm{C}=\mathrm{O}$, coumarin), $1610(\mathrm{C}=\mathrm{N}), 1712$ (stretching band $>\mathrm{C}=\mathrm{O}, \alpha, \beta$-unsaturated cinnamamide), $1548(-\mathrm{NH}), 638(\mathrm{C}-\mathrm{S}) .{ }^{1} \mathrm{H}$ NMR [400MHz, $\delta$, ppm, DMSO- $d_{6}$ ]: 7.19-7.93(m, 7H, ArH), 6.9-7.0(s, 1H, CH=CH), $5.25(\mathrm{~s}, 1 \mathrm{H},-$ $\mathrm{NH}), 4.17(\mathrm{~d}, 1 \mathrm{H},-\mathrm{OH})$. Anal. Calcd. for $\mathrm{C}_{21} \mathrm{H}_{14} \mathrm{~N}_{2} \mathrm{O}_{4} \mathrm{~S}$ : C, 64.61; H, 3.58; N, 7.17. Found: C, 64.87; $\mathrm{H}, 3.59 ; \mathrm{N}, 7.77 \%$.

\section{General procedure of 2,3-dihydro-2-aryl-4-[4-(2-oxo-2H-chromen-3-yl)-1,3-thiazol-2- ylamino]-1,5-benzothiazipine $(6 \mathbf{6}-\mathbf{j})^{17}$}

(a) Conventional method. In a $250 \mathrm{~mL}$ round-bottomed flask, 4a-j (0.01 mol), 2-aminothiophenol 5 and glacial acetic acid $(5 \mathrm{ml})$ in methanol were taken. The reaction mixture was refluxed on a water bath at $65-70{ }^{\circ} \mathrm{C}$ for $6-9$ hours and the solvent was distilled off. Then it was poured into crushed ice and the product filtered off, washed with water and recrystallized from ethanol.

(b) Microwave method. In a $250 \mathrm{~mL}$ round-bottomed flask, 4a-j (0.01 mol), 2-aminothiophenol 5 and glacial acetic acid $(5 \mathrm{ml})$ in methanol was taken. The reaction mixture was irradiated inside a "Q-Pro-M Modified Microwave" System at 300 Watt for 2.0-3.5 minutes. Then it was poured into crushed ice and the product filtered off, washed with water and recrystallized from ethanol to give 6 a.

2,3-Dihydro-2-(3-methoxy-4-hydroxyphenyl)-4-[4-(2-oxo-2H-chromen-3-yl)-1,3-thiazol-2ylamino]-1,5-benzothiazepine (6a). M.p. $119-120^{\circ} \mathrm{C}$; IR $\left[\mathrm{v}, \mathrm{cm}^{-1}, \mathrm{KBr}\right]: 3450(\mathrm{OH}), 3261 \mathrm{C}-\mathrm{H}$, aliphatic), 3027( $\mathrm{ArH}$, aromatic ring), 2830 $\left(\mathrm{OCH}_{3}\right), 1727(>\mathrm{C}=\mathrm{O}$, coumarin $), 1607(\mathrm{C}=\mathrm{N}), 1540(\mathrm{NH})$, 633(C-S). ${ }^{1} \mathrm{H}$ NMR [400MHz, $\delta$, ppm, DMSO-d 6 ]: 6.65-7.77(m,6H,ArH), 6.19(s,1H,-CH of thiazole), 5.21(s,1H,-NH), 4.11(d,1H,-OH), $\left.3.93\left(\mathrm{~d}, 2 \mathrm{H},-\mathrm{CH}_{2}, \mathrm{~J}=6.8 \mathrm{~Hz}\right)\right), 3.63\left(\mathrm{~d}, 3 \mathrm{H},-\mathrm{OCH}_{3}\right)$, $3.30(\mathrm{t}, 1 \mathrm{H},-\mathrm{CH}, \mathrm{J}=7.0 \mathrm{~Hz}) .{ }^{13} \mathrm{C} \mathrm{NMR}\left[100 \mathrm{MHz}, \delta, \mathrm{ppm}, \mathrm{DMSO}-d_{6}\right]^{17}: 168.29\left(\mathrm{C}_{13}\right), 125.37-$ 168.28( $\mathrm{C}_{17}, \mathrm{C}_{18}, \mathrm{C}_{19}, \mathrm{C}_{20}, \mathrm{C}_{21}, \mathrm{C}_{24}, \mathrm{C}_{25}, \mathrm{C}_{26}, \mathrm{C}_{27}$, heteroaromatics $), \quad 159.47\left(\mathrm{C}_{12}\right), \quad 158.11\left(\mathrm{C}_{7}\right)$, 
154.25( $\left(\mathrm{C}_{1}, \mathrm{C}_{2}\right), \quad 153.60\left(\mathrm{C}_{10}\right), \quad 152.33\left(\mathrm{C}_{22}, \mathrm{C}_{23}\right), \quad 135.22\left(\mathrm{C}_{11}\right), \quad 133.08\left(\mathrm{C}_{9}\right), \quad 116.25-125.30\left(\mathrm{C}_{3}, \mathrm{C}_{4}\right.$, $\mathrm{C}_{5}, \mathrm{C}_{6}$, aromatics $), 125.09\left(\mathrm{C}_{16}\right), 123.07\left(\mathrm{C}_{14}\right), 110.08\left(\mathrm{C}_{8}\right), 60.67\left(\mathrm{C}_{15}\right), 35.77\left(-\mathrm{OCH}_{3}\right) . \mathrm{MS}: \mathrm{m} / \mathrm{z}$ [528] ${ }^{+}$. Anal. Calcd. for $\mathrm{C}_{28} \mathrm{H}_{21} \mathrm{~N}_{3} \mathrm{O}_{4} \mathrm{~S}_{2}$ (527.6): C, 63.71; H, 4.98; N, 7.93. Found: C, 63.52; H, 4.02; N, 7.83\%.

2,3-Dihydro-2-(4-methylphenyl)-4-[4-(2-oxo-2H-chromen-3-yl)-1,3-thiazol-2-ylamino]-1,5benzothiazepine (6b). M.p. $110-111{ }^{\circ} \mathrm{C}$; IR $\left[\mathrm{v}, \mathrm{cm}^{-1}, \mathrm{KBr}\right]: 3270(\mathrm{C}-\mathrm{H}$, aliphatic), 3027(ArH, aromatic ring), 2923 $\left(\mathrm{CH}_{3}\right), 1722\left(>\mathrm{C}=\mathrm{O}\right.$, coumarin), 1609(C=N), 1542(NH), 632(C-S). ${ }^{1} \mathrm{H}$ NMR [400MHz, $\delta$, ppm, DMSO- $d_{6}$ ]: 6.73-7.87(m, 7H, ArH), $6.20(\mathrm{~s}, 1 \mathrm{H},-\mathrm{CH}$ of thiazole), $5.23(\mathrm{~s}, 1 \mathrm{H},-$ $\mathrm{NH}), 3.95\left(\mathrm{~d}, 2 \mathrm{H},-\mathrm{CH}_{2}, \mathrm{~J}=6.8 \mathrm{~Hz}\right), 3.32(\mathrm{t}, 1 \mathrm{H},-\mathrm{CH}, \mathrm{J}=7.0 \mathrm{~Hz}), 2.63\left(\mathrm{~s}, 3 \mathrm{H},-\mathrm{CH}_{3}\right) .{ }^{13} \mathrm{C} \mathrm{NMR}[100$ $\mathrm{MHz}, \delta$, ppm, DMSO-d $\left.d_{6}\right]$ : 167.98( $\left(\mathrm{C}_{13}\right), 114.87-167.18\left(\mathrm{C}_{17}, \mathrm{C}_{18}, \mathrm{C}_{19}, \mathrm{C}_{20}, \mathrm{C}_{21}, \mathrm{C}_{24}, \mathrm{C}_{25}, \mathrm{C}_{26}, \mathrm{C}_{27}\right)$, 158.86( $\left.\mathrm{C}_{12}\right), 157.88\left(\mathrm{C}_{7}\right), 153.71\left(\mathrm{C}_{1}, \mathrm{C}_{2}\right), 152.77\left(\mathrm{C}_{10}\right), 152.33\left(\mathrm{C}_{22}, \mathrm{C}_{23}\right), 134.49\left(\mathrm{C}_{11}\right), 132.78\left(\mathrm{C}_{9}\right)$, 116.25-131.80( $\left.\mathrm{C}_{3}, \mathrm{C}_{4}, \mathrm{C}_{5}, \mathrm{C}_{6}\right), 124.18\left(\mathrm{C}_{16}\right), 122.19\left(\mathrm{C}_{14}\right), 109.78\left(\mathrm{C}_{8}\right), 59.67\left(\mathrm{C}_{15}\right), 24.02\left(-\mathrm{CH}_{3}\right) . \mathrm{MS}$ : $\mathrm{m} / \mathrm{z}$ [496] $]^{+}$. Anal. Calcd. for $\mathrm{C}_{28} \mathrm{H}_{21} \mathrm{~N}_{3} \mathrm{O}_{3} \mathrm{~S}_{2}$ (495.6): C, 67.82; H, 4.29; N, 8.45. Found: C, 67.64; H, $4.31 ; \mathrm{N}, 8.37 \%$.

2,3-Dihydro-2-(2-chlorophenyl)-4-[4-(2-oxo-2H-chromen-3-yl)-1,3-thiazol-2-ylamino]-1,5-

benzothiazepine (6c). M.p. 121-122 ${ }^{\circ} \mathrm{C}$; IR $\left[\mathrm{v}, \mathrm{cm}^{-1}, \mathrm{KBr}\right]: 3270(\mathrm{C}-\mathrm{H}$, aliphatic), 3027(ArH, aromatic ring), $1722\left(>\mathrm{C}=\mathrm{O}\right.$, coumarin), 1609(C=N), 1542(NH), 811(C-Cl), 632(C-S). ${ }^{1} \mathrm{H}$ NMR [400MHz, $\delta, \mathrm{ppm}, \mathrm{DMSO}-d_{6}$ ]: 6.98-7.93(m,7H,ArH), 6.22(s,1H,-CH of thiazole), 5.20(s,1H,-NH), $3.96\left(\mathrm{~d}, 2 \mathrm{H},-\mathrm{CH}_{2}, \mathrm{~J}=6.8 \mathrm{~Hz}\right), 3.33(\mathrm{t}, 1 \mathrm{H},-\mathrm{CH}, \mathrm{J}=7.0 \mathrm{~Hz}) .{ }^{13} \mathrm{C} \mathrm{NMR}\left[100 \mathrm{MHz}, \delta, \mathrm{ppm}, \mathrm{DMSO}-d_{6}\right]$ : 169.28( $\left.\mathrm{C}_{13}\right), \quad 116.37-169.18\left(\mathrm{C}_{17}, \mathrm{C}_{18}, \mathrm{C}_{19}, \mathrm{C}_{20}, \mathrm{C}_{21}, \mathrm{C}_{24}, \mathrm{C}_{25}, \mathrm{C}_{26}, \mathrm{C}_{27}\right), \quad 160.47\left(\mathrm{C}_{12}\right), \quad 159.12 \quad\left(\mathrm{C}_{7}\right)$, $155.75\left(\mathrm{C}_{1}, \mathrm{C}_{2}\right), \quad 154.60\left(\mathrm{C}_{10}\right), \quad 153.33\left(\mathrm{C}_{22}, \mathrm{C}_{23}\right), \quad 139.19\left(\mathrm{C}_{11}\right), \quad 135.08\left(\mathrm{C}_{9}\right), \quad 117.25-130.19\left(\mathrm{C}_{3}\right.$, $\left.\mathrm{C}_{4}, \mathrm{C}_{5}, \mathrm{C}_{6}\right), 127.18\left(\mathrm{C}_{16}\right), 125.07\left(\mathrm{C}_{14}\right), 112.18\left(\mathrm{C}_{8}\right), 65.77\left(\mathrm{C}_{15}\right) . \mathrm{MS}: \mathrm{m} / \mathrm{z}[516]^{+}$. Anal. Calcd. for $\mathrm{C}_{27} \mathrm{H}_{18} \mathrm{~N}_{3} \mathrm{O}_{2} \mathrm{~S}_{2} \mathrm{Cl}$ (516.0): C, 62.82; H, 3.49; N, 8.17. Found: $\mathrm{C}, 62.73 ; \mathrm{H}, 3.41 ; \mathrm{N}, 8.11 \%$.

2,3-Dihydro-2-(4-chlorophenyl)-4-[4-(2-oxo-2H-chromen-3-yl)-1,3-thiazol-2-ylamino]-1,5-

benzothiazepine (6d). M.p. 127-128 ${ }^{\circ} \mathrm{C}$; IR [v, cm $\left.{ }^{-1}, \mathrm{KBr}\right]: 3270(\mathrm{C}-\mathrm{H}$, aliphatic), 3027(ArH, aromatic ring), $1722\left(>\mathrm{C}=\mathrm{O}\right.$, coumarin), 1609(C=N), 1542(NH), 814(C-Cl), 632(C-S). ${ }^{1} \mathrm{H}$ NMR [400MHz, $\delta$, ppm, DMSO-d $]$ ]: 7.00-7.95(m, 7H, ArH), 6.23(s,1H,-CH of thiazole), 5.19(s,1H,-NH), $3.95\left(\mathrm{~d}, 2 \mathrm{H},-\mathrm{CH}_{2}, \mathrm{~J}=6.8 \mathrm{~Hz}\right), 3.31(\mathrm{t}, 1 \mathrm{H},-\mathrm{CH}, \mathrm{J}=7.0 \mathrm{~Hz}),{ }^{13} \mathrm{C} \mathrm{NMR}\left[100 \mathrm{MHz}, \delta\right.$, ppm, DMSO- $\left.d_{6}\right]$ : 168.88( $\left(\mathrm{C}_{13}\right), \quad 115.77-168.68\left(\mathrm{C}_{17}, \mathrm{C}_{18}, \mathrm{C}_{19}, \mathrm{C}_{20}, \mathrm{C}_{21}, \mathrm{C}_{24}, \mathrm{C}_{25}, \mathrm{C}_{26}, \mathrm{C}_{27}\right), \quad 159.67\left(\mathrm{C}_{12}\right), \quad 158.11 \quad\left(\mathrm{C}_{7}\right)$, $154.75\left(\mathrm{C}_{1}, \mathrm{C}_{2}\right), \quad 154.57\left(\mathrm{C}_{10}\right), \quad 152.63\left(\mathrm{C}_{22}, \mathrm{C}_{23}\right), \quad 134.18\left(\mathrm{C}_{11}\right), \quad 134.18\left(\mathrm{C}_{9}\right), \quad 116.25-129.90\left(\mathrm{C}_{3}\right.$, $\left.\mathrm{C}_{4}, \mathrm{C}_{5}, \mathrm{C}_{6}\right), 125.93\left(\mathrm{C}_{16}\right), 123.27\left(\mathrm{C}_{14}\right), 111.08\left(\mathrm{C}_{8}\right), 60.17\left(\mathrm{C}_{15}\right) . \mathrm{MS}: \mathrm{m} / \mathrm{z}$ [516] ${ }^{+}$. Anal. Calcd. for $\mathrm{C}_{27} \mathrm{H}_{18} \mathrm{~N}_{3} \mathrm{O}_{2} \mathrm{~S}_{2} \mathrm{Cl}$ (516.0): C, 62.82; H, 3.49; N, 8.17. Found: C, 62.77; H, 3.39; N, 8.07\%.

2,3-Dihydro-2-(2,4-dichlorophenyl)-4-[4-(2-oxo-2H-chromen-3-yl)-1,3-thiazol-2-ylamino]-1,5benzothiazepine (6e). M.p. 129-130 ${ }^{\circ} \mathrm{C}$; IR $\left[\mathrm{v}, \mathrm{cm}^{-1}, \mathrm{KBr}\right]: 3270(\mathrm{C}-\mathrm{H}$, aliphatic), 3027(ArH, aromatic ring), $1722\left(>\mathrm{C}=\mathrm{O}\right.$, coumarin), $1609(\mathrm{C}=\mathrm{N}), 1542(\mathrm{NH}), 820(\mathrm{C}-\mathrm{Cl}), 632(\mathrm{C}-\mathrm{S}) .{ }^{1} \mathrm{H}$ NMR [400MHz, $\delta$, ppm, DMSO-d 6 ]: 6.78-7.86(m, 6H, Ar-H), 6.21(s,1H,-CH of thiazole $), 5.19(\mathrm{~s}, 1 \mathrm{H},-$ $\mathrm{NH}), 3.97\left(\mathrm{~d}, 2 \mathrm{H},-\mathrm{CH}_{2}, \mathrm{~J}=6.8 \mathrm{~Hz}\right), 3.32(\mathrm{t}, 1 \mathrm{H},-\mathrm{CH}, \mathrm{J}=7.0 \mathrm{~Hz}) .{ }^{13} \mathrm{C} \mathrm{NMR}[100 \mathrm{MHz}, \delta$, ppm, DMSO$d_{6}$ : $168.97\left(\mathrm{C}_{13}\right), 115.45-168.87\left(\mathrm{C}_{17}, \mathrm{C}_{18}, \mathrm{C}_{19}, \mathrm{C}_{20}, \mathrm{C}_{21}, \mathrm{C}_{24}, \mathrm{C}_{25}, \mathrm{C}_{26}, \mathrm{C}_{27}\right), 159.87\left(\mathrm{C}_{12}\right), 158.11\left(\mathrm{C}_{7}\right)$, 154.25( $\left.\mathrm{C}_{1}, \mathrm{C}_{2}\right), \quad 153.50\left(\mathrm{C}_{10}\right), \quad 152.87\left(\mathrm{C}_{22}, \mathrm{C}_{23}\right), \quad 135.12\left(\mathrm{C}_{11}\right), \quad 133.78\left(\mathrm{C}_{9}\right), \quad 116.25-129.90\left(\mathrm{C}_{3}\right.$, $\left.\mathrm{C}_{4}, \mathrm{C}_{5}, \mathrm{C}_{6}\right), 125.45\left(\mathrm{C}_{16}\right), 123.19\left(\mathrm{C}_{14}\right), 111.08\left(\mathrm{C}_{8}\right), 60.56\left(\mathrm{C}_{15}\right) . \mathrm{MS}: \mathrm{m} / \mathrm{z}[550]^{+}$. Anal. Calcd. for $\mathrm{C}_{27} \mathrm{H}_{17} \mathrm{~N}_{3} \mathrm{O}_{2} \mathrm{~S}_{2} \mathrm{Cl}_{2}$ (550.4): C, 58.94; H, 3.13; N, 7.61. Found: C, 58.82; H, 3.11; N, 7.51\%. 
2,3-Dihydro-2-(2-methoxyphenyl)-4-[4-(2-oxo-2H-chromen-3-yl)-1,3-thiazol-2-ylamino]-1,5benzothiazepine (6f). M.p. $137-138{ }^{\circ} \mathrm{C}$; IR $\left[\mathrm{v}, \mathrm{cm}^{-1}, \mathrm{KBr}\right]: 3270(\mathrm{C}-\mathrm{H}$, aliphatic), 3027(ArH, aromatic ring), 2828 $\left(\mathrm{OCH}_{3}\right), 1722\left(>\mathrm{C}=\mathrm{O}\right.$, coumarin), $1609(\mathrm{C}=\mathrm{N}), 1542(\mathrm{NH}), 632(\mathrm{C}-\mathrm{S}) .{ }^{1} \mathrm{H}$ NMR [400MHz, $\delta$, ppm, DMSO- $d_{6}$ ]: 6.72-7.82(m, 7H, ArH), 6.22(s,1H,-CH of thiazole), $5.22(\mathrm{~s}, 1 \mathrm{H},-$ $\mathrm{NH}), 3.94\left(\mathrm{~d}, 2 \mathrm{H},-\mathrm{CH}_{2}, \mathrm{~J}=6.8 \mathrm{~Hz}\right), 3.63\left(\mathrm{~s}, 3 \mathrm{H},-\mathrm{OCH}_{3}\right), 3.34(\mathrm{t}, 1 \mathrm{H},-\mathrm{CH}, \mathrm{J}=7.0 \mathrm{~Hz}) .{ }^{13} \mathrm{C} \mathrm{NMR}[100$ $\mathrm{MHz}, \delta$, ppm, DMSO-d $\left.d_{6}\right]: 168.98\left(\mathrm{C}_{13}\right), 115.89-168.65\left(\mathrm{C}_{17}, \mathrm{C}_{18}, \mathrm{C}_{19}, \mathrm{C}_{20}, \mathrm{C}_{21}, \mathrm{C}_{24}, \mathrm{C}_{25}, \mathrm{C}_{26}, \mathrm{C}_{27}\right)$, 159.79( $\left.\mathrm{C}_{12}\right), 158.19\left(\mathrm{C}_{7}\right), 154.46\left(\mathrm{C}_{1}, \mathrm{C}_{2}\right), 153.46\left(\mathrm{C}_{10}\right), 152.56\left(\mathrm{C}_{22}, \mathrm{C}_{23}\right), 135.45\left(\mathrm{C}_{11}\right), 134.08\left(\mathrm{C}_{9}\right)$, 114.25-129.90( $\left.\mathrm{C}_{3}, \mathrm{C}_{4}, \mathrm{C}_{5}, \mathrm{C}_{6}\right), 125.12\left(\mathrm{C}_{16}\right), 123.46\left(\mathrm{C}_{14}\right), 110.18\left(\mathrm{C}_{8}\right), 60.78\left(\mathrm{C}_{15}\right), 36.71\left(-\mathrm{OCH}_{3}\right)$. MS: m/z [512] ; Anal. Calcd. for $\mathrm{C}_{28} \mathrm{H}_{21} \mathrm{~N}_{3} \mathrm{O}_{3} \mathrm{~S}_{2}$ (550.4): C, 65.75; H, 4.17; N, 8.18. Found: C, $65.71 ; \mathrm{H}, 4.11 ; \mathrm{N}, 8.10 \%$.

\section{2,3-Dihydro-2-(4-methoxyphenyl)-4-[4-(2-oxo-2H-chromen-3-yl)-1,3-thiazol-2-ylamino]-1,5-}

benzothiazepine (6g). M.p. 131-132 ${ }^{\circ} \mathrm{C}$; IR $\left[\mathrm{v}, \mathrm{cm}^{-1}, \mathrm{KBr}\right]: 3270(\mathrm{C}-\mathrm{H}$, aliphatic), 3027(ArH, aromatic ring), 2830 $\left(\mathrm{OCH}_{3}\right), 1722\left(>\mathrm{C}=\mathrm{O}\right.$, coumarin), $1609(\mathrm{C}=\mathrm{N}), 1542(\mathrm{NH}), 632(\mathrm{C}-\mathrm{S}) .{ }^{1} \mathrm{H}$ NMR [400MHz, $\delta$, ppm, DMSO- $d_{6}$ ]: 6.70-7.81(m,7H, ArH), 6.25(s,1H,-CH of thiazole), $5.20(\mathrm{~s}, 1 \mathrm{H},-\mathrm{NH})$, $3.96(\mathrm{t}, 1 \mathrm{H},-\mathrm{CH}, \mathrm{J}=7.0 \mathrm{~Hz}), 3.63\left(\mathrm{~d}, 2 \mathrm{H},-\mathrm{CH}_{2}, \mathrm{~J}=6.8 \mathrm{~Hz}\right), 3.29\left(\mathrm{~d}, 3 \mathrm{H},-\mathrm{OCH}_{3}\right) .{ }^{13} \mathrm{C} \mathrm{NMR}[100 \mathrm{MHz}, \delta$, ppm, DMSO- $\left.d_{6}\right]: 168.84\left(\mathrm{C}_{13}\right), 115.85-168.86\left(\mathrm{C}_{17}, \mathrm{C}_{18}, \mathrm{C}_{19}, \mathrm{C}_{20}, \mathrm{C}_{21}, \mathrm{C}_{24}, \mathrm{C}_{25}, \mathrm{C}_{26}, \mathrm{C}_{27}\right), 159.78\left(\mathrm{C}_{12}\right)$, $158.11\left(\mathrm{C}_{7}\right), \quad 154.57\left(\mathrm{C}_{1}, \mathrm{C}_{2}\right), \quad 153.06\left(\mathrm{C}_{10}\right), \quad 152.77\left(\mathrm{C}_{22}, \mathrm{C}_{23}\right), \quad 135.22\left(\mathrm{C}_{11}\right), \quad 133.80\left(\mathrm{C}_{9}\right), \quad 116.52-$ 129.09( $\left.\mathrm{C}_{3}, \mathrm{C}_{4}, \mathrm{C}_{5}, \mathrm{C}_{6}\right), 125.65\left(\mathrm{C}_{16}\right), 123.71\left(\mathrm{C}_{14}\right), 110.80\left(\mathrm{C}_{8}\right), 60.76\left(\mathrm{C}_{15}\right), 34.77\left(\mathrm{OCH}_{3}\right) . \mathrm{MS}: \mathrm{m} / \mathrm{z}$ [512] ; Anal. Calcd. for $\mathrm{C}_{28} \mathrm{H}_{21} \mathrm{~N}_{3} \mathrm{O}_{3} \mathrm{~S}_{2}$ (511.6): C, 65.71; H, 4.11; N, 8.91. Found: C, 65.64; $\mathrm{H}$, 4.07; N, 8.71\%.

2,3-Dihydro-2-(3,4,5-trimethoxyphenyl)-4-[4-(2-oxo-2H-chromen-3-yl)-1,3-thiazol-2-ylamino]1,5-benzothiazepine (6h). M.p. $135-136{ }^{\circ} \mathrm{C}$; IR $\left[\mathrm{v}, \mathrm{cm}^{-1}, \mathrm{KBr}\right]: 3270(\mathrm{C}-\mathrm{H}$, aliphatic), 3027(ArH, aromatic ring), 2835( $\left.\mathrm{OCH}_{3}\right), 1722\left(>\mathrm{C}=\mathrm{O}\right.$, coumarin), $1609(\mathrm{C}=\mathrm{N}), 1542(\mathrm{NH}), 632(\mathrm{C}-\mathrm{S}) .{ }^{1} \mathrm{H}$ NMR [400MHz, $\left.\delta, \mathrm{ppm}, \mathrm{DMSO}-d_{6}\right]: 6.73-7.89(\mathrm{~m}, 7 \mathrm{H}, \mathrm{ArH}), 6.22(\mathrm{~s}, 1 \mathrm{H},-\mathrm{CH}$ of thiazole $), 5.19(\mathrm{~s}, 1 \mathrm{H},-\mathrm{NH})$, $3.97\left(\mathrm{~d}, 2 \mathrm{H},-\mathrm{CH}_{2}, \mathrm{~J}=6.8 \mathrm{~Hz}\right), 3.63\left(\mathrm{~s}, 3 \mathrm{H},-\mathrm{OCH}_{3}\right), 3.31(\mathrm{t}, 1 \mathrm{H},-\mathrm{CH}, \mathrm{J}=7.0 \mathrm{~Hz}) .{ }^{13} \mathrm{C} \mathrm{NMR}[100 \mathrm{MHz}, \delta$, ppm, DMSO-d $d_{6}$ : 168.95( $\left.\mathrm{C}_{13}\right), 115.89-167.99\left(\mathrm{C}_{17}, \mathrm{C}_{18}, \mathrm{C}_{19}, \mathrm{C}_{20}, \mathrm{C}_{21}, \mathrm{C}_{24}, \mathrm{C}_{25}, \mathrm{C}_{26}, \mathrm{C}_{27}\right), 159.98\left(\mathrm{C}_{12}\right)$, $158.99\left(\mathrm{C}_{7}\right), \quad 155.25\left(\mathrm{C}_{1}, \mathrm{C}_{2}\right), \quad 153.79\left(\mathrm{C}_{10}\right), \quad 152.79\left(\mathrm{C}_{22}, \mathrm{C}_{23}\right), 135.56\left(\mathrm{C}_{11}\right), 133.79\left(\mathrm{C}_{9}\right), 116.23-$ 129.19( $\left.\mathrm{C}_{3}, \mathrm{C}_{4}, \mathrm{C}_{5}, \mathrm{C}_{6}\right), 126.03\left(\mathrm{C}_{16}\right), 123.85\left(\mathrm{C}_{14}\right), 110.77\left(\mathrm{C}_{8}\right), 61.67\left(\mathrm{C}_{15}\right), 35.4\left(\mathrm{OCH}_{3}\right) ; \mathrm{MS}: \mathrm{m} / \mathrm{z}$ [572] ; Anal. Calcd. for $\mathrm{C}_{30} \mathrm{H}_{25} \mathrm{~N}_{3} \mathrm{O}_{5} \mathrm{~S}_{2}$ (571.6): C, 63.06; H, 4.39; N, 7.38. Found: C, 63.04; H, 4.30; N, 7.29\%.

2,3-Dihydro-2-(4- $N, N$-dimethylaminophenyl)-4-[4-(2-oxo-2H-chromen-3-yl)-1,3-thiazol-2-yl

amino]-1,5-benzothiazepine (6i). M.p. $138-139{ }^{\circ} \mathrm{C}$; IR [v, $\left.\mathrm{cm}^{-1}, \mathrm{KBr}\right]: 3270(\mathrm{C}-\mathrm{H}$, aliphatic), 3027 (ArH, aromatic ring), 1722 $\left(>\mathrm{C}=\mathrm{O}\right.$, coumarin), $\left.1609(\mathrm{C}=\mathrm{N}), 1542(\mathrm{NH}), 1315 \mathrm{~N}\left(-\mathrm{CH}_{3}\right)_{2}\right), 632 \quad$ (CS). ${ }^{1} \mathrm{H}$ NMR [400MHz, $\delta$, ppm, DMSO- $\left.d_{6}\right]: 6.71-7.83(\mathrm{~m}, 7 \mathrm{H}, \mathrm{ArH}), 6.23(\mathrm{~s}, 1 \mathrm{H},-\mathrm{CH}$ of thiazole), 5.20(s,1H,-NH), 3.98(d,2H,-CH $2, \quad J=6.8 H z), 3.30(t, 1 \mathrm{H},-\mathrm{CH}, \mathrm{J}=7.0 \mathrm{~Hz}), 2.9\left(\mathrm{~m}, 6 \mathrm{H},-\mathrm{N}\left(\mathrm{CH}_{3}\right)_{2}\right) .{ }^{13} \mathrm{C}$ NMR[100MHz, $\delta, \quad$ ppm, DMSO- $\left.d_{6}\right]: \quad 168.86\left(\mathrm{C}_{13}\right), \quad 115.54-168.86\left(\mathrm{C}_{17}, \mathrm{C}_{18}, \mathrm{C}_{19}, \mathrm{C}_{20}\right.$, $\left.\mathrm{C}_{21}, \mathrm{C}_{24}, \mathrm{C}_{25}, \mathrm{C}_{26}, \mathrm{C}_{27}\right), \quad 159.95\left(\mathrm{C}_{12}\right), \quad 158.85\left(\mathrm{C}_{7}\right), \quad 154.59\left(\mathrm{C}_{1}, \mathrm{C}_{2}\right), \quad 153.35\left(\mathrm{C}_{10}\right), \quad 152.25\left(\mathrm{C}_{22}, \mathrm{C}_{23}\right)$, $135.35\left(\mathrm{C}_{11}\right), \quad 133.33\left(\mathrm{C}_{9}\right), \quad 117.59-129.91\left(\mathrm{C}_{3}, \mathrm{C}_{4}, \mathrm{C}_{5}, \mathrm{C}_{6}\right), \quad 125.58\left(\mathrm{C}_{16}\right), \quad 123.32\left(\mathrm{C}_{14}\right), \quad 110.10\left(\mathrm{C}_{8}\right)$, 60.06( $\left(\mathrm{C}_{15}\right), 21.41\left(\mathrm{CH}_{3}\right)$; MS: m/z [524] ; Anal. Calcd. for $\mathrm{C}_{29} \mathrm{H}_{24} \mathrm{~N}_{4} \mathrm{O}_{3} \mathrm{~S}_{2}(524.6): \mathrm{C}, 66.36 ; \mathrm{H}, 4.63$; N, 10.66. Found: C, 66.28; H, 4.52; N, 10.59\%. 


\section{2,3-Dihydro-2-(4-hydroxyphenyl)-4-[4-(2-oxo-2H-chromen-3-yl)-1,3-thiazol-2-ylamino]-1,5-}

benzothiazepine (6j). M.p. $117-118^{\circ} \mathrm{C}$; IR [ $\left.\mathrm{v}, \mathrm{cm}^{-1}, \mathrm{KBr}\right]: 3460(-\mathrm{OH}), 3027(\mathrm{ArH}$, aromatic ring), 1722 $\left(>\mathrm{C}=\mathrm{O}\right.$, coumarin), $1609(\mathrm{C}=\mathrm{N}), 1542(-\mathrm{NH}), 3270\left(\mathrm{C}-\mathrm{H}\right.$, aliphatic), 632(C-S). ${ }^{1} \mathrm{H}$ NMR [400MHz, $\delta, \mathrm{ppm}$, DMSO- $d_{6}$ ]: 6.85-7.65(m,7H,ArH $), 6.22(\mathrm{~s}, 1 \mathrm{H},-\mathrm{CH}$ of thiazole), $5.21(\mathrm{~s}, 1 \mathrm{H},-\mathrm{NH})$, $4.11(\mathrm{~s}, 1 \mathrm{H},-\mathrm{OH}), 3.97\left(\mathrm{~d}, 2 \mathrm{H},-\mathrm{CH}_{2}, \mathrm{~J}=6.8 \mathrm{~Hz}\right), 3.32(\mathrm{t}, 1 \mathrm{H},-\mathrm{CH}, \mathrm{J}=7.0 \mathrm{~Hz}) .{ }^{13} \mathrm{C}$ NMR $(100 \mathrm{MHz}, \delta, \mathrm{ppm}$, DMSO- $\left.d_{6}\right): \quad 168.86\left(\mathrm{C}_{13}\right), \quad 115.37-168.88\left(\mathrm{C}_{17}, \mathrm{C}_{18}, \mathrm{C}_{19}, \mathrm{C}_{20}, \mathrm{C}_{21}, \mathrm{C}_{24}, \mathrm{C}_{25}, \mathrm{C}_{26}, \mathrm{C}_{27}\right), \quad 159.95\left(\mathrm{C}_{12}\right)$, $158.85\left(\mathrm{C}_{7}\right), \quad 154.45\left(\mathrm{C}_{1}, \mathrm{C}_{2}\right), \quad 153.39\left(\mathrm{C}_{10}\right), \quad 152.93\left(\mathrm{C}_{22}, \mathrm{C}_{23}\right) \quad 135.99\left(\mathrm{C}_{11}\right), 133.39\left(\mathrm{C}_{9}\right), 116.16-$ 129.92( $\left.\mathrm{C}_{3}, \mathrm{C}_{4}, \mathrm{C}_{5}, \mathrm{C}_{6}\right), 125.83\left(\mathrm{C}_{16}\right), 123.77\left(\mathrm{C}_{14}\right), 110.11\left(\mathrm{C}_{8}\right), 60.97\left(\mathrm{C}_{15}\right) . \mathrm{MS}: \mathrm{m} / \mathrm{z}[479]^{+}$; Anal. Calcd. for $\mathrm{C}_{27} \mathrm{H}_{19} \mathrm{~N}_{3} \mathrm{O}_{3} \mathrm{~S}_{2}$ (479.5): C, 62.55; H, 3.27; N, 10.41. Found: C, 62.43; H, 3.18; N, 10.07\%.

\section{Acknowledgements}

The authors are thankful to Veer Narmad South Gujarat University, Surat (Gujarat) for providing research facilities. One of the authors (JTD) is also thankful to Dr. Jignesh R. Desai, Gharda Chemicals Ltd., Dombivali, Thane (Maharastra) and SAIF, Punjab University, Chandigrah (India) for providing spectral and analytical data of the compounds $\mathbf{4 a - j}$ and $\mathbf{6 a - j}$. The authors also thanks to the Head of Bioscience Department of VNSGU, Surat for biological screening.

\section{References}

1. Bigi, F. Tetrahedron Lett. 2001, 42, 5203.

2. Warad, D. U.; Satish, C. D.; Kulkarni, V. H.; Bajgur, C. S. Indian J Chem. 2000, 39A, 415.

3. Handan, A.; Oznur, A.; K. Ayse, Seher, B.; Gulten, O. Indian. J. Chem. 2005, 44B, 585.

4. Xie, L.; Tukeuchi, Y.; Consetino, L. M.; Lee, K. J. Med. Chem. 1999, 42, 2662.

5. Desai, H. K.; Gawad, D. H.; Joshi, B. S. Indian J. Chem. 1977, 15 B, 291.

6. Buckel, D. R.; Smith, H. J. Med. Chem. 1975, 18, 391.

7. Karal, B. K.; Chavan, V. P.; Mame, A. S.; Hangarage, R. V. Korean J. Med. Chem. 2000, 10, 84; Chem. Abstr. 2001, 134, 147581j.

8. Kinoshita, K.; Mitani, A.; Hearse, J. D.; Braimbridge, V. M.; Manning, S. H. J. Surg. Res. 1989, 47, 166; Chem. Abstr. 1989, 111, 126689k.

9. Belusa, J.; Hruskova, V.; Haas, Z.; Kaminska, Z.; Picha, F.; Dusek, J.; Trefulka, M.; Kysilka, V.; Wojnar, V. Czech, CS 274,213,1992; Chem. Abstr. 1992, 118, 2459706.

10. Padwad, M.; Ingle, V. N. J. Indian Chem. Soc. 1999, 76, 161.

11. Nikalje, A. G.; Ingle, R. D.; Bhingolikar, V. E.; Mane, K. A.; Indian J. Heterocycl. Chem. 2003, $13,33$.

12. Upreti, M.; Pant, S.; Dandia, A.; Pant, U.C. Indian J. Chem. 1997, 36B, 1181.

13. Kuzelova, M.; Svec, P. Cesk. Farm. 1993, 42, 124; Chem. Abstr. 1994, 120, 45561w.

14. (a) Pant, S.; Sharma, B. S.; Sharma, C. K.; Pant, U. C. J. Indian. Chem. Soc. 1996, 73, 83. (b) Pant, U. C.; Singhal, B.; Pant, S. Indian J. Heterocycl. Chem. 2001, 10, 185. 
15. Desai, A. R.; Desai, K. R. ARKIVOC 2005, (xiii), 98.

16. Patel, V. M.; Desai, K. R. ARKIVOC 2004, (i), 123.

17. Mistry, K. M.; Desai, K. R. J. Soudi Chem. Soc. 2005, 9, 381.

18. Katritzky, A. R.; Singh, S. K. ARKIVOC 2003, (xiii), 68.

19. Raval, J. P.; Desai, K. R. ARKIVOC 2005, (xiii), 21.

20. Raval, J. P.; Desai K. G.; Desai, K. R. J. Iranian Chem. Soc. 2006, 3, 233.

21. Desai K. G.; Desai, K. R. Bioorg. Med. Chem. 2006, 14, 8271.

22. Desai K. G.; Desai, K. R. J. Heterocycl. Chem. 2006, 43, 1.

23. Desai, A. R.; Desai, K. R. J. Heterocycl. Chem. 2005, 42, 995.

24. Mistry, K. M.; Desai, K. R. Indian J. Chem. 2005, 44B, 1452.

25. (a) Gradwol's Clinical Laboratory methods and diagnosis. $7^{\text {th }}$ Edition, Mosby, C.V. Company: Germany, 1970; Vol. II, p1407. (b) National Committee for Clinical Laboratory Standards. Reference method for broth dilution antifungal susceptibility testing of yeast. Approved standard NCCLS document M27-A. (ISBN 1-56238-328-0, ISSN 0273-3099). National Committee for Clinical Laboratory Standards, 940, West Valley Road, Suite 1400, Wayne, Pennsylvania, 19807), 1997.

26. (a) Sahu, S. K.; Mishra, A.; Behera, R. K. Indian J. Heterocycl. Chem. 1996, 6, 91. (b) Naik, B. N.; Desai, K. R. Indian J. Chem. 2006, 45, 267.

27. Desai, J. T.; Desai, C. K.; Desai K. R. J. Iranian Chem. Soc. 2006, 5, 67. 